\title{
DER ÖSTERREICHISCHE BIBLIOTHEKENVERBUND IM UMBRUCH: NEUES BIBLIOTHEKSVERWALTUNGSSYSTEM ALMA ALS IMPULS FÜR DIE ZUKUNFT
}

von Bruno Bauer, Markus Lackner und Bernhard Schubert

Zusammenfassung: Die aktuelle Ausgabe der „Mitteilungen der Vereinigung Österreichischer Bibliothekarinnen und Bibliothekare" (VÖB) ist dem Schwerpunktthema Implementierung von Alma im Österreichischen Bibliothekenverbund gewidmet.

Schlüsselwörter: Österreichischer Bibliothekenverbund; OBV; Österreichische Bibliotheken- und Service GmbH; Bibliotheksverwaltungssystem; Aleph 500; Alma; Systemwechsel; Editorial

\section{THE AUSTRIAN LIBRARY NETWORK IN TRANSITION: THE NEW INTEGRATED LIBRARY SYSTEM ALMA AS AN IMPULSE FOR THE FUTURE}

Abstract: The current issue of the „Mitteilungen der Vereinigung Österreichischer Bibliothekarinnen und Bibliothekare "(VÖB)/,,Communications of the Association of Austrian Librarians" is dedicated to the implementation of Alma in the Austrian Library Network.

Keywords: Austrian Library Network; ALN; Austrian Library Network and Service Ltd; integrated library system; Aleph 500; Alma; ILS change; editorial

DOI: https://doi.org/10.31263/voebm.v71i2.2130

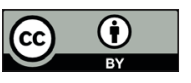


bereits in Ausgabe 2/2011 der Mitteilungen der VÖB wurde mit dem Schwerpunktthema "Suchmaschinentechnologie an wissenschaftlichen Bibliotheken" ein wichtiges Verbundthema in den Fokus unserer Zeitschrift gerückt. Auch die aktuelle Schwerpunktausgabe 2/2018 der Mitteilungen der VÖB ist mit „Alma im Österreichischen Bibliothekenverbund" einem Thema gewidmet, das den Österreichischen Bibliothekenverbund in seiner Gesamtheit, aber auch viele der Verbundbibliotheken in der jüngsten Vergangenheit sehr stark dominiert hat: der Einführung eines neuen Bibliotheksverwaltungssystems.

Während bei der letzten Systemablösung die magische Datumsgrenze 1999/2000 ein wesentlicher Impuls für den Wechsel von BIBOS zu Aleph 500 gewesen war (Stichwort „Systemgrenze“)2, so standen bei der aktuellen Ablösung von Aleph 500 durch Alma ${ }^{3}$, die 2013 gestartet worden war und die 2017 für die ersten Bibliotheken erfolgreich abgeschlossen werden konnte, große Erwartungen für wichtige neue Tools im Fokus, insbesondere für den Bereich der elektronischen Ressourcen und der statistischen Auswertungen. Weitere Herausforderungen waren die Annäherung an ein Cloud-System, die mit der DSGVO einhergehenden Anforderungen (inklusive des damit zusammenhängenden Themas Betriebsvereinbarungen) sowie die Durchführung eines aufwändigen Vergabeverfahrens.

Die vergangenen Jahre können auch als „Krise“ für den Österreichischen Bibliothekenverbund beschrieben werden - im Sinn des aus dem Griechischen stammenden Substantivs zum altgriechischen Verb „krínein“, welches „trennen“ und „(unter-)scheiden“ bedeutet. Krise bezeichnet gemäß Duden „(Ent-)Scheidung“, „entscheidende Wendung“ und bedeutet "schwierige Situation, Zeit, die den Höhe- und Wendepunkt einer gefährlichen Entwicklung darstellt". Exakt diese Entwicklung wurde auch beim Wechsel von Aleph 500 zu Alma gemacht, weil zunächst stark divergierende Interessen - fertiges System einer kommerziellen Firma versus Open Source, lokales Hosting versus Cloud-System - evident wurden. Diese mündeten allerdings dank der Kompromissfähigkeit aller beteiligten Institutionen in einer gemeinsamen und nachhaltigen Lösung. Es ist festzuhalten, dass der Österreichische Bibliothekenverbund gestärkt aus dem Vergabeverfahren und der Implementierungsphase des neuen Systems herausgegangen ist und sich fit für die kommenden Herausforderungen des nächsten Jahrzehnts präsentiert.

Mit dem erfolgreich koordinierten und federführend umgesetzten Systemwechsel hat auch die Verbundzentrale eindrucksvoll unter Beweis gestellt, dass sie eine wichtige und verlässliche Partnerin für alle teilneh- 
menden Einrichtungen ist. Deren Aufgabenportfolio sollte neben den traditionellen Verbundaufgaben, die im Errichtungsgesetz der OBVSG $(2002)^{4}$ und in den Leistungsvereinbarungen der OBVSG mit dem Bundesministerium für Bildung, Wissenschaft und Kultur $(2005)^{5}$ angeführt sind und deren Überprüfung und Anpassung an die aktuellen Gegebenheiten sinnvoll und notwendig wäre, auch wichtige Zukunftsfelder - von der Langzeitarchivierung bis zum Betrieb von Infrastruktur für Open Access, Open Educational Resources und Forschungsdaten - umfassen.

\section{Meilensteine des Systemwechsels Aleph 500 / Alma im OBV}

\section{9}

- Erstmalige Thematisierung der Aleph-Ablöse in der AG Strategische Planung im Österreichischen Bibliothekenverbund

\section{0-2013}

- Einrichtung einer AG Aleph-Ablöse durch die Verbund-Vollversammlung für die Marktsondierung, die Ermittlung von Anforderungen sowie die Erstellung eines Pflichtenheftes für das zukünftige Bibliotheksverwaltungssystem im Österreichischen Bibliothekenverbund

\section{3}

- Start des Vergabeverfahrens durch Bekanntmachung der „Beschaffung eines Bibliothekenverbundsystems mit lokalen, zentralen und konsortialen Funktionalitäten" durch die 14 Auftraggeber im Supplement zum Amtsblatt der Europäischen Union

\section{3-2015}

- Durchführung eines Verhandlungsverfahrens und Zuschlag der 14 Auftraggeberinstitutionen im Österreichischen Bibliothekenverbund für das Produkt Alma der Bestbieterfirma Ex Libris

\section{6-2018}

- Umsetzung der Aleph-Ablöse und der Implemenierung des neuen Bibliotheksverwaltungssystems Alma an den 14 Auftraggeberinstitutionen in drei Phasen:

Konzeptionsphase - Implementierungsphase - Inbetriebnahme 
In der aktuellen Schwerpunktausgabe wird das Thema „Alma im Österreichischen Bibliothekenverbund" in vier Beiträgen, zwei Reports und einem Interview dargestellt. Die beteiligten Autorinnen und Autoren bringen dabei aus unterschiedlichen Perspektiven ihre Sicht auf dieses große, zukunftsweisende Projekt ein, wobei zum Teil eher der Blick auf die unmittelbare Vergangenheit, zum Teil eher der Blick auf die Zukunft im Vordergrund steht. Ergänzt wird der Alma-Schwerpunkt durch eine Mitteilung und einen Veranstaltungsbericht.

\section{Beiträge}

Markus Lackner beschreibt „Die Alma-Implementierung im Österreichischen Bibliothekenverbund (OBV) unter besonderer Berücksichtigung der Kohorte 2-Bibliotheken". Nachdem die Implementierung für Kohorte 1 zwischen Dezember 2016 und September 2017 erfolgreich umgesetzt werden konnte, folgte Kohorte 2 zwischen Juli 2017 und Jänner 2018, die Verbundzentrale zwischen Dezember 2017 und März 2018. Im Beitrag werden Struktur, Konzeption und Zeitplan des Gesamtprojekts im Österreichischen Bibliothekenverbund sowie die Umsetzung der Implementierung an den sieben Kohorte 2-Bibliotheken vorgestellt [S. 267].

Im Beitrag „Von Alephino nach Alma, ein Dramolett in drei Akten: Die AlmaImplementierung im Österreichischen Bibliothekenverbund am Beispiel der Bibliothek der Fachhochschule Sankt Pölten" geben Christian Kieslinger und Karl Rathmanner Einblick in die Erfahrungen der weltweit ersten Institution, die von Alephino nach Alma migriert ist [S. 276].

Bernhard Schubert, Christian Beiler, Christoph Steiner, Rainer Steltzer und Petra Gratzl befassen sich eingehend mit „Erschließungsarbeit in Alma“ und thematisieren dabei „Erfahrungen aus dem OBV vor, während und nach der Aleph-Ablöse". Besonderes Augenmerk wird auf die Auswirkungen des Umstiegs auf die konkrete (Formal- und Sach-)Erschließungsarbeit gelegt [S. 282].

„Alma im Österreichischen Bibliothekenverbund (OBV): Aus der Werkstatt der OBVSG" lautet der Titel des Beitrags von Bettina Kann, in dem sie die neue Verbundarchitektur mit dem Parallelbetrieb zwischen Alma und Aleph 500 vorstellt. Begleitet wird dieses Übergangskonzept von neuen Schlagwörtern, wie MAB-MARC-Konverter, Kreiskonvertierung und Kreishybridisierung. Weiters erläutert Kann die Umstellung der verschiedenen zentralen Services, wie Primo, GND, Visual Library, IV-Scan und SAP-Anbindung, auf diese neue Architektur [S. 307]. 


\section{Reports}

Eine besonders wichtige Rolle bei Vorbereitung und Umsetzung des Systemwechsels kam auch den Expertinnen und Experten an den einzelnen Bibliotheken zu, die in acht Gruppen (Daten, E-Ressourcen, Entlehnung, Erwerbung, Fernleihe, Katalogisierung, Öffentliche Services, Zeitschriften) sowie einer Schulungsgruppe als Schnittstelle zur OBVSG und zur Herstellerfirma fungiert haben. Im Report „Implementierung des neuen Bibliotheksmanagementsystems Alma an 14 Einrichtungen im Österreichischen Bibliothekenverbund - Feedback aus der Perspektive der Functional Experts" haben sie ihre Erfahrungen anhand von jeweils vier Fragen zusammengefasst: (1) Was ist bei der Implementierung von Alma für Daten gut gelaufen? (2) Wo traten beim Implementierungsprozess Schwächen auf? (3) Was sollte man beim nächsten Wechsel des Bibliothekssystems unbedingt anders bzw. besser machen? (4) Welche Wünsche und Erwartungen für die künftige Entwicklung von Alma gibt es? [S. 320].

„Wo gehest Du hin? Zukünftige Dienstleistungen der OBVSG - Ergebnisse einer Umfrage" - unter diesem Titel skizziert Robert Schiller, welche zukünftigen Anforderungen, Bedürfnisse und Wünsche im Rahmen einer von der AG "Strategische Planung“ 2017 durchgeführten Umfrage an den Bibliotheken im Österreichischen Bibliothekenverbund erhoben worden sind. Ziel dieser Erhebung war es, eine Grundlage zu schaffen, die es der Österreichischen Bibliothekenverbund und Service GmbH (OBVSG) ermöglicht, eine strategische Anpassung ihres Dienstleistungsportfolios vorzunehmen [S. 351].

\section{Interview}

Abgeschlossen wird die Schwerpunktausgabe mit einem Interview zum Thema „Alma im österreichischen Bibliothekenverbund und die Perspektiven für die Österreichische Bibliothekenverbund und Service GmbH: Wolfgang Hamedinger, Geschäftsführer der OBVSG, beantwortet 10 Fragen von Bruno Bauer". Der Fokus des Interviews liegt auf den Rahmenbedingungen für die im Österreichischen Bibliothekenverbund 2017 erfolgte Umstellung vom bisherigen Bibliotheksverwaltungssystem Aleph 500 auf das neue System Alma und auf den besonderen Herausforderungen, die dieses Projekt gekennzeichnet haben, das als dominierendes Thema die Entwicklung des wissenschaftlichen Bibliothekswesens in Österreich mehr als ein Jahrzehnt lang geprägt hat. Thematisiert werden auch weitere Geschäftsfelder der OBVSG sowie deren Finanzierung und die Frage nach der Zukunft von Bibliotheken und Bibliotheksverbünden [S. 356]. 


\section{Mitteilung}

Mathis Kronschläger informiert in den „Mitteilungen aus der OBVSG“ über den Go-Live der Alma Network Zone und die aus diesem Grund veranstaltete „Alma Gleichenfeier“, die am 20. Juni 2018 in Wien stattgefunden hat, über den Beitritt der Universitätsbibliothek der Wirtschaftsuniversität Wien zur ZDB im Österreichischen Bibliothekenverbund und über die neu bestellte Leitung der Abteilung „Laufende Planung / Implementierung“ an der OBVSG [S. 371].

\section{Veranstaltungsbericht}

Ebenfalls von Mathis Kronschläger erstellt wurde der Bericht über den 14. Verbundtag, der am 15. Mai an der Alpen-Adria-Universität Klagenfurt stattgefunden hat. Dieser wurde geprägt vom Systemwechsel von Aleph auf Alma und dem nur wenige Wochen zurückliegenden Go-Live der Alma Network Zone [S. 377].

Mag. Bruno Bauer

ORCID: http://orcid.org/0000-0002-4729-331X Medizinische Universität Wien, Universitätsbibliothek

E-Mail: bruno.bauer@meduniwien.ac.at

Dipl.-Ing. (FH) Markus Lackner Universität Graz, Universitätsbibliothek

E-Mail: markus.lackner@uni-graz.at

Mag. Bernhard Schubert, BA MSc ORCID: http://orcid.org/0000-0003-1748-9087 Universität Wien, Bibliotheks- und Archivwesen

E-Mail: bernhard.schubert@univie.ac.at

1 Schwerpunktthema "Suchmaschinentechnologie an wissenschaftlichen Bibliotheken". In: Mitteilungen der Vereinigung Österreichischer Bibliothekarinnen \& Bibliothekare 64 (2011), H. 2. Online: https://phaidra. univie.ac.at/ o:103633/

2 Wolfgang Hamedinger: Der große Wechsel. Von Bibos zu Aleph 500. In: Mitteilungen der Vereinigung Österreichischer Bibliothekarinnen \& Bibliothekare 52 (1999), H. 1, S. 38-42. Online: http://www.literature. 
at $/$ viewer.alo? objid=14011\&viewmode=fullscreen\&scale=2.5\&rotate $=$ \&page $=40$

3 Wolfgang Hamedinger: Austrian Library Network and Next Generation Library System: Alma. In: BIBLIOTHEK - Forschung und Praxis 40 (2016), H. 3, S. 341-346. https://doi.org/10.1515/bfp-2016-0055

4 Bundesgesetz über die Österreichische Bibliothekenverbund und Service Gesellschaft mit beschränkter Haftung. In: Bundesgesetzblatt für die Republik Österreich, 08.01.2002. Online: https://www.ris.bka. gv.at/Dokumente/BgblPdf/2002_15_1/2002_15_1.pdf

5 Leistungsvereinbarung gemäß $\S 4$ Abs. 3 des Bundesgesetzes über die Österreichische Bibliothekenverbund und Service Gesellschaft m.b.H., BGBI. I Nr. 15/2002. Unterfertigt am 15.03.2005. Online: https:// www.vbk.ac.at/files/docs_recht/public/Leistungsvereinbarung\%20 OBVSG\%202002.pdf 